\title{
Smeared BTZ Black Hole from Space Noncommutativity
}

\author{
Hyeong-Chan $\mathrm{Kim}^{a, *}$, Mu-In Park ${ }^{b, \dagger}$, Chaiho Rim ${ }^{b, \ddagger}$, and Jae Hyung Yee ${ }^{a, \S}$ \\ ${ }^{a}$ Korea Department of Physics, Yonsei University, Seoul 120-749, Republic of Korea \\ ${ }^{a}$ Department of Physics and Research Institute of Physics and Chemistry, \\ Chonbuk National University, Chonju 561-756, Korea
}

\begin{abstract}
We study a novel phenomena of smearing of black hole horizons from the effect of space noncommutativity. We present an explicit example in $A d S_{3}$ space, using the Chern-Simons formulation of gravity. This produces a smeared BTZ black hole which goes beyond the classical spacetime unexpectedly and there is no reality problem in our approach with the gauge group $U(1,1) \times U(1,1)$. The horizons are smeared, due to a splitting of the Killing horizon and the apparent horizon, and there is a metric signature change to Euclidean in the smeared region. The inner boundary of the smeared region acts as a trapped surface for timelike particles but the outer as a classical barrier for ingoing particles. The lightlike signals can escape from or reach the smeared region in a finite time, which indicates that the black hole is not so dark, even classically. In addition, it is remarked that the Hawking temperature can not be defined by the regularity in the Euclidean geometry except in the non-rotating case, and the origin can be smeared by a new (apparent) horizon.
\end{abstract}

PACS numbers: 04.70.Dy, 04.60.Kz, 11.10.Nx

\footnotetext{
* E-mail address: hckim@phya.yonsei.ac.kr

$\dagger$ E-mail address: muinpark@gmail.com

$\ddagger$ E-mail address: rim@chonbuk.ac.kr

$\S$ E-mail address: jhyee@phya.yonsei.ac.kr
} 


\section{INTRODUCTION}

It is known that noncommutative field theories [1, 2] modify the short distance behaviors of conventional theories. For example, the noncommutativities can smooth out a singularity of classical solutions in the conventional theories [3]. However, similar modifications in gravity theories are not studied much, though there are several formulations of noncommutative gravities and speculations. For example, one can generally expect a "smearing" of the horizons, which are sharply defined in the conventional spacetime. From the commutation relations

$$
\left[x^{i}, x^{j}\right]=i \theta^{i j}
$$

where $\theta^{i j}$ is an antisymmetric constant of dimension lengt $h^{2}$, the precise location of a horizon is limited by the uncertainty relations $\Delta x^{i} \Delta x^{j} \geq\left|\theta^{i j}\right| / 2$. But, there has been no explicit demonstration of existence of the smearing region with unexpected space-time structure.

In this paper, we investigate the modifications of the BTZ black hole in three-dimensional anti-de Sitter $\left(A d S_{3}\right)$ space with a polar form of the commutation relation

$$
[r, \phi]=i \hat{\theta}
$$

for a spherically symmetric case $\left(\theta^{r \phi} \equiv \hat{\theta}\right)^{1}$, using the Chern-Simons formulation of gravity. This is the first explicit example of the novel phenomena of the horizon smearing which goes beyond the classical spacetime unexpectedly, and without the reality problem by considering the gauge group $U(1,1) \times U(1,1)$. It is found that the event horizon is smeared by the splitting of the Killing horizon and the apparent horizon with the thickness of the order $\hat{\theta}$ and the smeared horizon acts as a classical barrier for particles. The time duration of light signals escaping from and reaching the smeared horizon region from outside is demonstrated to be finite. The physical effect of the metric signature change inside the smeared region is considered. In addition, it is remarked that the Hawking temperature from the regularity in the Euclidean geometry is not applicable, and the naked conical singularity at the origin in $A d S_{3}$ or negative mass solutions, generally, is smeared due to the presence of a "new" apparent horizon near the origin.

\section{THREE-DIMENSIONAL NONCOMMUTATIVE GRAVITY FROM CHERN- SIMONS FORMULATION}

It is well known that, in three-dimensional space-time, conventional gravity theory can be formulated as a Chern-Simons theory [5]. This provides a novel way to define the noncommutative gravity theory [6]. An explicit solution in the noncommutative gravity can be obtained from the corresponding known solution in the commutative gravity, via the Seiberg-Witten map [7].

\footnotetext{
${ }^{1}$ This commutation relations differ from the Cartesian ones with a constant $\theta^{i j}$ [4] since it corresponds to a non-constant $\theta^{i j}=r \hat{\theta} \epsilon^{i j}$ in (1). But, the Moyal product [1] can be still consistently defined in the polar coordinate with a constant $\theta^{r \phi}$. And we expect no big difference in the qualitative physics near the horizon since we are considering physics at $r \neq 0$.
} 
The $(2+1)$-dimensional noncommutative gravity with the negative cosmological constant $\Lambda=-1 / l^{2}$ is defined by the $U(1,1) \times U(1,1)$ Chern-Simons theory, up to surface terms,

$$
I_{g}[\hat{\mathcal{A}}]=\frac{l}{16 \pi G} \int \operatorname{Tr}\left(\hat{\mathcal{A}}^{+} \star d \hat{\mathcal{A}}^{+}+\frac{2}{3} \hat{\mathcal{A}}^{+} \star \hat{\mathcal{A}}^{+} \star \hat{\mathcal{A}}^{+}\right)-\left(\hat{\mathcal{A}}^{+} \leftrightarrow \hat{\mathcal{A}}^{-}\right) .
$$

(Here, the wedge symbol has been omitted.) The connections are given by ${ }^{2}$

$$
\hat{\mathcal{A}}^{ \pm}=\left(\hat{\omega}^{a} \pm \hat{e}^{a} / l\right) t_{a}+i \hat{c}^{ \pm} \mathbf{1}
$$

with the triads and the $S U(1,1)$ spin connections $\hat{e}^{a}=\hat{e}_{\mu}^{a} d x^{\mu}, \hat{\omega}_{\mu}^{a}=(1 / 2) \epsilon^{a b c} \hat{\omega}_{\mu b c} d x^{\mu}(a=$ $0,1,2)$, respectively, and the $U(1)$ connections $c^{ \pm}$which make the group closed with respects to the Moyal $\star$ product $[1,6]$

$$
\star=\exp \left[\frac{i}{2} \hat{\theta}\left(\overleftarrow{\partial_{r}} \overrightarrow{\partial_{\phi}}-\overleftarrow{\partial_{\phi}} \overrightarrow{\partial_{r}}\right)\right]
$$

Here, it is important to note that, in the commutative limit, the theory does not depend on the metric, i.e., the choice of the coordinates $[8]^{3}$. In the polar coordinates $\mu=(t, r, \phi)$, for example, the (commutative) action takes the form [9]: $I_{g}[A]=$ $(l / 16 \pi G) \int d t d r d \phi \operatorname{Tr}\left(-A_{r}^{+} \partial_{t} A_{\phi}^{+}+A_{\phi}^{+} \partial_{t} A_{r}^{+}+2 A_{t}^{+} F_{r \phi}^{+}\right)-\left(A_{\mu}^{+} \leftrightarrow A_{\mu}^{-}\right)$. We consider the Moyal $\star$ deformation of this polar action as follows:

$$
I_{g}[\hat{\mathcal{A}}]=\frac{l}{16 \pi G} \int d t d r d \phi \operatorname{Tr}\left(-\hat{\mathcal{A}}_{r}^{+} \star \partial_{t} \hat{\mathcal{A}}_{\phi}^{+}+\hat{\mathcal{A}}_{\phi}^{+} \star \partial_{t} \hat{\mathcal{A}}_{r}^{+}+2 \hat{\mathcal{A}}_{t}^{+} \star \hat{\mathcal{F}}_{r \phi}^{+}\right)-\left(\hat{\mathcal{A}}_{\mu}^{+} \leftrightarrow \hat{\mathcal{A}}_{\mu}^{-}\right)
$$

(We define $\hat{\mathcal{F}}_{\mu \nu}^{ \pm}=\partial_{\mu} \hat{\mathcal{A}}_{\nu}^{ \pm}+\hat{\mathcal{A}}_{\mu}^{ \pm} \star \hat{\mathcal{A}}_{\nu}^{ \pm}-(\mu \leftrightarrow \nu)$, in a covariant way.) We note that the noncommutative action has the measure of integration $d t d r d \phi$ and so the Moyal product (5) is well defined, as in the usual Cartesian coordinates [7]. There are some differences in the global properties of the coordinates, due to the range of the coordinates $(0, \infty) \times$ $(0,2 \pi)$ and so there will be some appropriate boundary conditions on the allowed functions (see footnote 4, for example). However all the standard "local" (i.e., ignoring boundary conditions) formula in Cartesian coordinates, like equations of motion and the SeibergWitten map, will work also here [].

The equations of motion are given by

$$
d \hat{\mathcal{A}}^{ \pm}+\hat{\mathcal{A}}^{ \pm} \star \hat{\mathcal{A}}^{ \pm}=0
$$

which are not easy to solve directly. But using the Seiberg-Witten map [10], which transforms the noncommutative Chern-Simons action into the commutative one, or vice versa ${ }^{4}$,

${ }^{2}$ We take the $S U(1,1)$ bases $t_{0}=\sigma_{2} / 2, t_{1}=i \sigma_{3} / 2, t_{2}=\sigma_{1} / 2$ such that $\left[t_{a}, t_{b}\right]=-\epsilon_{a b}^{c} t_{c}, \operatorname{Tr}\left(t_{a} t_{b}\right)=(1 / 2) \tilde{\eta}_{a b}$ with $\epsilon_{012}=1$ and $\tilde{\eta}_{a b}=\operatorname{diag}(+1,-1,+1)$.

${ }^{3}$ The metric dependence in the Cartesian measure of integration, $d^{3} x$, under the general coordinate transformations, $d^{3} x^{\prime}=d^{3} x / \sqrt{g}$, is canceled by that of the Levi-Civita tensor density $\epsilon^{\alpha \beta \gamma}=$ $\sqrt{g} \partial_{\mu} x^{\prime \alpha} \partial_{\nu} x^{\prime \beta} \partial_{\rho} x^{\prime \gamma} \epsilon^{\mu \nu \rho}$ in the Chern-Simons 3-form $d^{3} x \epsilon^{\mu \nu \rho} \operatorname{Tr}\left[A_{\mu} \partial_{\nu} A_{\rho}+(2 / 3) A_{\mu} A_{\nu} A_{\rho}\right]$.

${ }^{4}$ In this mapping, there are several boundary terms which do not vanish for an arbitrary choice of $\theta^{i j}$, generally. But, these terms vanish for our choice (2) and the commutative solutions $\mathcal{A}^{ \pm}$which decrease rapidly for large $r$. 
without additional action [7], any solution $\mathcal{A}^{ \pm}$of the commutative equations $d \mathcal{A}^{ \pm}+\mathcal{A}^{ \pm} \mathcal{A}^{ \pm}=$ 0 can be mapped into the corresponding $\hat{\mathcal{A}}^{ \pm}$of the noncommutative equations (17) [11]:

$$
\hat{\mathcal{A}}_{\mu}^{ \pm}(\theta)=\mathcal{A}_{\mu}^{ \pm}+(i / 4) \theta^{\alpha \beta}\left[\mathcal{A}_{\alpha}^{ \pm}, \partial_{\beta} \mathcal{A}_{\mu}^{ \pm}+\mathcal{F}_{\beta \mu}^{ \pm}\right]_{+}+\mathcal{O}\left(\theta^{2}\right) .
$$

From the obtained solution $\hat{\mathcal{A}}^{ \pm}$, one can compute $\hat{\omega}$ and $\hat{e}$ which describe the noncommutative gravity.

\section{BTZ BLACK HOLE SOLUTION WITH U(1) FLUXES IN $U(1,1) \times U(1,1)$ CHERN-SIMONS GRAVITY}

In the commutative limit, the equations of motion reduce to the two sets of decoupled equations

$$
\begin{aligned}
& d \omega+\omega \omega-\left(1 / l^{2}\right) e e=0, d e+\omega e+e \omega=0, \\
& d c^{ \pm}=0 .
\end{aligned}
$$

We generalize these to the case with the non-vanishing $U(1)$ fluxes $d c^{ \pm}=f^{ \pm}$by adding an additional term $-2 \int \operatorname{Tr}(f \mathcal{A})$ to the Chern-Simons gravity action. This modifications do not change the conventional gravity equations (9), since these are decoupled from the $U(1)$-parts (10).

In the noncommutative case, we have the additional noncommutative action $-2 \int \operatorname{Tr}(\hat{f} \star$ $\hat{\mathcal{A}}$ ) [12]. One can confirm that this term is invariant under the Seiberg-Witten map, for the appropriate fluxes $\hat{f}$ which decrease rapidly for large $r$ with our choice (2). In consequence, the solution $\hat{\mathcal{A}}$ of the generalized theory can be simply obtained by the same mapping (8) from the corresponding commutative solution $\mathcal{A}$. The $U(1)$ fields are not decoupled anymore and have non-trivial effects on the commutative gravity solutions.

To proceed, we consider the Aharonov-Bohm type $U(1)$ potentials

$$
c^{ \pm}=\Phi^{ \pm} d \phi
$$

which give the fluxes inside the horizons, $f^{ \pm}=2 \pi \Phi^{ \pm} \delta^{2}(\mathbf{x}) d r d \phi$. For the (commutative) gravity solution, we consider the BTZ black hole given by [13]

$$
d s^{2}=-N^{2} d t^{2}+N^{-2} d r^{2}+r^{2}\left(d \phi+N^{\phi} d t\right)^{2}
$$

with $N^{2}=\left(r^{2}-r_{+}^{2}\right)\left(r^{2}-r_{-}^{2}\right) / l^{2} r^{2}, N^{\phi}=-r_{+} r_{-} / l r^{2}$. Here, $r_{+}$and $r_{-}$denote the outer and inner horizons, respectively. The $S U(1,1) \times S U(1,1)$-form gauge connections $\mathbf{A}^{ \pm}=$ $\left(\omega^{a} \pm e^{a} / l\right) t_{a}$ are given by [14]

$$
\mathbf{A}^{ \pm}=\frac{1}{2}\left(\begin{array}{cc} 
\pm i(l / \nu) d \mu & z_{ \pm}(\nu \mp i \mu)(d t / l \mp d \phi) \\
z_{ \pm}(\nu \pm i \mu)(d t / l \mp d \phi) & \mp i(l / \nu) d \mu
\end{array}\right),
$$

where $\nu^{2}(r)=\left(r^{2}-r_{-}^{2}\right) /\left(r_{+}^{2}-r_{-}^{2}\right), \mu^{2}=\nu^{2}-1$, and $z_{ \pm}=\left(r_{+} \pm r_{-}\right) / l$.

For the noncommutativity relations (2) with $\hat{\theta}$ rescaled as $\hat{\theta}=l \theta$ and others $=0$, the solutions $\hat{\mathcal{A}}^{ \pm}$in the noncommutative gravity ([6) are obtained, via the Seiberg-Witten map,

$$
\begin{aligned}
& \hat{\mathcal{A}}_{t}^{ \pm}=i(l \theta / 4) \operatorname{Tr}\left(A_{\phi}^{ \pm} \partial_{r} A_{t}^{ \pm}\right) \mathbf{1}+\left[1+(l \theta / 2) c_{\phi}^{ \pm} \partial_{r}\right] A_{t}^{ \pm}, \\
& \hat{\mathcal{A}}_{r}^{ \pm}=i(l \theta / 4) \operatorname{Tr}\left(A_{\phi}^{ \pm} \partial_{r} A_{r}^{ \pm}\right) \mathbf{1}+\left[1+(l \theta / 2)\left\{\left(\partial_{r} c_{\phi}^{ \pm}\right)+c_{\phi}^{ \pm} \partial_{r}\right\}\right] A_{r}^{ \pm}, \\
& \hat{\mathcal{A}}_{\phi}^{ \pm}=\left[1+(l \theta / 2)\left(\partial_{r} c_{\phi}^{ \pm}\right)\right] \mathcal{A}_{\phi}^{ \pm},
\end{aligned}
$$


(note that $\mathcal{A}_{t, r}^{ \pm}=A_{t, r}^{ \pm}, \mathcal{A}_{\phi}^{ \pm}=c_{\phi}^{ \pm}+A_{\phi}^{ \pm}$), neglecting the higher order terms of $\mathcal{O}\left(\theta^{2}\right)$. The metric of the noncommutative gravity is defined ${ }^{5}$ as $d \hat{s}^{2}=\eta_{a b}\left(\hat{e}_{\mu}^{a} \star \hat{e}_{\nu}^{b}\right) d x^{\mu} d x^{\nu}$ with $\eta_{a b}=$ $\operatorname{diag}(-1,+1,+1)$ and given by

$$
d \hat{s}^{2}=-f^{2} d t^{2}+\hat{N}^{-2} d r^{2}+r^{2}\left(d \phi+N^{\phi} d t\right)^{2}+\mathcal{O}\left(\theta^{2}\right),
$$

where

$$
\begin{aligned}
\hat{N}^{2} & =\frac{r^{2}}{l^{2}}+\theta c_{\phi} \frac{r}{l}-\frac{\left(r_{+}^{2}+r_{-}^{2}\right)}{l^{2}}+\frac{r_{+}^{2} r_{-}^{2}}{l^{2} r^{2}}-\theta c_{\phi} \frac{r_{+}^{2} r_{-}^{2}}{l r^{3}} \\
f^{2} & =\hat{N}^{2}+\theta c_{\phi} \frac{r_{+}^{2} r_{-}^{2}}{l r^{3}}
\end{aligned}
$$

Here, we consider $c_{\phi}^{+}=c_{\phi}^{-}$for simplicity and omit the singular term at the origin $-2 \pi l \theta N^{2} \Phi \delta^{2}(\mathbf{x})$ in $\hat{N}^{2}$ since we are considering physics at $r \neq 0$. Note that, in this case, the noncommutative spacetime satisfies the same gravity equations of motion as in the commutative limit, i.e., $d \hat{\omega}+\hat{\omega} \hat{\omega}-\left(1 / l^{2}\right) \hat{e} \hat{e}=0$, dê $+\hat{\omega} \hat{e}+\hat{e} \hat{\omega}=0$, with no Moyal products, from (7) and the triviality of bi-products in our solution (14), i.e., $\hat{\omega} \star \hat{\omega}=\hat{\omega} \hat{\omega}, \hat{\omega} \star \hat{e}=\hat{\omega} \hat{e}$, etc. In other words, the (first-order) noncommutative solution (14) has the same noncommutative curvature $\hat{R} \equiv d \hat{\omega}+\hat{\omega} \hat{\omega}=\left(1 / l^{2}\right) \hat{e} \hat{e}$ and (zero) torsion $\hat{T} \equiv d \hat{e}+\hat{\omega} \hat{e}+\hat{e} \hat{\omega}=0$, as in the conventional BTZ black hole spacetime, outside the point flux [14]. ${ }^{6}$

\section{PROPERTIES OF THE SMEARED BLACK HOLE}

The noncommutative black hole solution has several remarkable properties which go beyond the classical geometry, unexpectedly.

1. There is a splitting of the apparent horizon and the Killing horizon: The apparent horizon is defined as a null hypersurface $g^{\mu \nu}\left(\partial_{\mu} r\right)\left(\partial_{\nu} r\right)=\hat{N}^{2}=0$, whereas the Killing horizon as the surface where the norm of the Killing vector $\chi=\partial_{t}+\Omega_{H} \partial_{\phi}$ vanishes, i.e., $\chi^{2}=g_{t t}-\left(g_{t \phi}\right)^{2} / g_{\phi \phi}=-f^{2}=0$ with the angular velocity of the horizon $\Omega_{H}=-\left.\left(g_{t \phi} / g_{\phi \phi}\right)\right|_{H}$.

We first note that $\hat{N}^{2}=\left[x^{5}+\theta c_{\phi} x^{4}-\left(x_{+}^{2}+x_{-}^{2}\right) x^{3}+\left(x_{+}^{2} x_{-}^{2}\right) x-\theta c_{\phi} x_{+}^{2} x_{-}^{2}\right] / x^{3}=0(x \neq$ $0, x \equiv r / l)$ has the outer/inner (apparent) horizons at

$$
\hat{r}_{ \pm}=r_{ \pm}-l \theta c_{\phi} / 2
$$

\footnotetext{
${ }^{5}$ Here, the metric has no "reality problem" since the equality $\hat{e}_{\mu} \star \hat{e}_{\nu}=\hat{e}_{\mu} \hat{e}_{\nu}$ holds in our metric (12) due to the commutation relations (2). This is a unique feature of our approach and in sharply contrast to the previous approaches [15] where the metric becomes complex generally and some truncations are needed to get a real metric.

${ }^{6}$ This may be compared with a BTZ solution in the presence of higher derivative terms, like the gravitational Chern-Simons action term. There the BTZ solution satisfies, trivially, the Einstein's equations of motion, with no higher derivative contributions. However, this does not mean a triviality of the solution since its physical parameters, like the ADM mass and angular momentum are significantly deformed from the conventional ones [16].
} 
The apparent horizons are equally shifted by the small amount $-l \theta c_{\phi} / 2$. On the other hand, the outer/inner Killing horizons can be obtained from $f^{2}=\left[x^{4}+\theta c_{\phi} x^{3}-\left(x_{+}^{2}+x_{-}^{2}\right) x^{2}+\right.$ $\left.x_{+}^{2} x_{-}^{2}\right] / x^{2}=0$,

$$
\tilde{r}_{ \pm}=r_{ \pm}-\left(l \theta c_{\phi} / 2\right)\left(1-r_{\mp}^{2} / r_{ \pm}^{2}\right)^{-1} .
$$

This shows that Killing horizons are not equally shifted so that the apparent and Killing horizons do not coincide each other in general, except in the non-rotating BTZ $\left(r_{-}=0\right)$ 7. It is noted that the solution (19) of the Killing horizons are not valid near the extremal commutative black holes with $r_{+}=r_{-}$where the higher order corrections are needed in contrast to the apparent horizons (18).

2. The event horizon becomes "smeared", due to the splitting of the Killing and apparent horizons: To see this, let us consider the metric

$$
d \hat{s}^{2}=-f^{2} d t^{2}+\hat{N}^{-2} d r^{2}+r^{2}\left(d \tilde{\phi}+\tilde{N}^{\phi} d t\right)^{2}
$$

such as $\tilde{N}^{\phi}=0$ either at the Killing horizons $\tilde{r}_{+}$or the apparent horizon $\hat{r}_{+}$, for an appropriate choice of the co-rotating frame. Then, the radial null geodesics are given by

$$
d r / d t= \pm \sqrt{f^{2} \hat{N}^{2}}
$$

with the upper (lower) sign corresponding to outgoing (ingoing) geodesics.

Near the Killing horizon $\tilde{r}_{+}$, the radial null geodesics for $r>\tilde{r}_{+}$are given by

$$
d r / d t= \pm \sqrt{2 \widetilde{\kappa}\left(-\theta c_{\phi}\right) r_{+}^{2} r_{-}^{2} / l \tilde{r}_{+}^{3}} \sqrt{r-\tilde{r}_{+}},
$$

where $\tilde{\kappa}$ corresponds to the surface gravity in the usual context $\left(f^{2} \approx 2 \tilde{\kappa}\left(r-\tilde{r}_{+}\right)\right)$:

$$
\begin{aligned}
\tilde{\kappa} & =\left.(1 / 2)\left(\partial f^{2} / \partial r\right)\right|_{\tilde{r}_{+}} \\
& =\left(\tilde{r}_{+}^{4}-r_{+}^{2} r_{-}^{2}\right) /\left(l^{2} \tilde{r}_{+}^{3}\right)+\theta c_{\phi} / 2 l .
\end{aligned}
$$

For the non-negative $\tilde{\kappa}$, which is always the case when $\theta$ is not so large, the outgoing as well as the ingoing geodesics are "classically" allowed only if the velocity (22) is real, i.e., $\theta c_{\phi}<0$, or $r_{+}<\hat{r}_{+}<\tilde{r}_{+}$.

Even though the light cones close up as we approach the horizon $\tilde{r}_{+}$, which signals usually that the time coordinate $t$ is badly defined near the horizon, it is remarkable that the light signals can escape from and reach the horizon $\tilde{r}_{+}$in a finite time

$$
\tilde{t} \approx\left[\tilde{\kappa}\left(-\theta c_{\phi}\right) r_{+}^{2} r_{-}^{2} / 2 l \tilde{r}_{+}^{3}\right]^{-1 / 2} \sqrt{r-\tilde{r}_{+}} .
$$

This is in contrast to the conventional commutative case ${ }^{8}$, where one needs an infinite time $t \sim \ln \left(r-r_{+}\right)$to escape from the horizon $r_{+}$though a finite "proper" time to reach the horizon [18]. It seems that the singular behavior of the time coordinate $t$ near the horizon is "moderated" by the noncommutativity effect. Thus, the horizon is smeared and not so "dark", even classically!

\footnotetext{
7 However, the inner horizons $\hat{r}_{-}, \tilde{r}_{-}$are absent in this case, though not manifest in (18) -(19).

8 Note that this behavior can not be directly obtained by setting $\theta c_{\phi}=0$ in (24) since we must consider $\left(r-r_{+}\right)$-term again which now dominates $\theta c_{\phi}$-term, in that case.
} 
The same is true when $\theta c_{\phi}>0$, or $\tilde{r}_{+}<\hat{r}_{+}<r_{+}$. In this case, the radial null geodesics for $r>\hat{r}_{+}$near the apparent horizon $\hat{r}_{+}$is given by

$$
d r / d t= \pm \sqrt{2 \hat{\kappa}\left(\theta c_{\phi}\right) r_{+}^{2} r_{-}^{2} / l \hat{r}_{+}^{3}} \sqrt{r-\hat{r}_{+}}
$$

where $\hat{N}^{2} \approx 2 \hat{\kappa}\left(r-\hat{r}_{+}\right)$and $f^{2} \approx\left(2 \hat{\kappa}-3 \theta c_{\phi} r_{+}^{2} r_{-}^{2} / l \hat{r}_{+}^{4}\right)\left(r-\hat{r}_{+}\right)+\theta c_{\phi} r_{+}^{2} r_{-}^{2} / l \hat{r}_{+}^{3}$ are used with

$$
\begin{aligned}
\hat{\kappa} & =\left.(1 / 2)\left(\partial \hat{N}^{2} / \partial r\right)\right|_{\hat{r}_{+}} \\
& =\left(\hat{r}_{+}^{4}-r_{+}^{2} r_{-}^{2}\right) /\left(l^{2} \hat{r}_{+}^{3}\right)+\theta c_{\phi} / 2 l+3 \theta c_{\phi} r_{+}^{2} r_{-}^{2} /\left(2 l \hat{r}_{+}^{4}\right) .
\end{aligned}
$$

The geodesics show that the escaping time from (or approaching time to) the horizon $\hat{r}_{+}$is finite

$$
\hat{t} \approx\left[\hat{\kappa}\left(\theta c_{\phi}\right) r_{+}^{2} r_{-}^{2} / 2 l \hat{r}_{+}^{3}\right]^{-1 / 2} \sqrt{r-\hat{r}_{+}} .
$$

3. The smeared horizon region behaves as a barrier for particles and waves: This comes from the fact that $f^{2} \hat{N}^{2}<0$ in the region between $\tilde{r}_{+}$and $\hat{r}_{+}$and one has the imaginary radial velocities for the (radial) null geodesics (21) ${ }^{9}$. This is the consequence of the fact that there is a "signature change" 10 to Euclidean $(+++)$, in the smeared region i.e., there is "no-time" and there are no light cones for the radial motions when $\theta c_{\phi}<0$. In this sense, the outer (horizon) boundary becomes hard to penetrate for particles, compared to the conventional event horizon. Nevertheless, light wave ${ }^{11}$ may tunnel the smeared horizon region when its wavelength is greater than the thickness of the region. (Similar thing happens when $\theta c_{\phi}>0$. In this case, we have (pseudo) Euclidean geometry with the signature $(--+)$, i.e., there are "two-times" in the smeared region, and time and radial coordinates change the role.)

However, particles or waves inside the inner boundary can not escape from the black hole since the light cone structures in that region are the same as in the interior region of the event horizon of the commutative case. So, the inner boundary of the smeared region is the trapped surface. The usual Hawking radiation would be generated near the inner boundary since one of the pair-created particles can be trapped. On the other hand, the pair-created particles near the outer boundary always recombine, due to the absence of the trapping. Thus, our result seems to favor the tunneling picture of the Hawking radiation by Parikh and Wilczek [18]. Further studies are needed in this direction.

4. The Hawking temperature defined by the periodicity in the Euclidean time is not applicable: To see this, let us consider the metric (20) near the Killing horizon $\tilde{r}_{+}$. Following the usual approach, we put the Euclidean time $\tau=-i t$ and we get

$$
d \hat{s}^{2} \approx \tilde{\kappa}^{2} \eta^{2} d \tau^{2}+\left[\left(1+\frac{3 \theta c_{\phi} r_{-}^{2}}{2 l r_{+}^{2} \tilde{\kappa}}\right)-\frac{\theta c_{\phi} r_{-}^{2}}{l r_{+} \tilde{\kappa}^{2} \eta^{2}}\right]^{-1} d \eta^{2}+\left(\tilde{r}_{+}+\tilde{\kappa} \eta^{2} / 2\right)^{2}(d \tilde{\phi})^{2}
$$

where $\eta=\sqrt{2\left(r-\tilde{r}_{+}\right)}$. From the regularity of the $d \tau^{2}$-part, one obtains the periodicity $\beta=2 \pi / \tilde{\kappa}$ which would give the conventional Hawking temperature $T_{H}=\tilde{\kappa} / 2 \pi[19]$. For the

9 This can be also observed in (22) and (25).

10 This seems to reflect the quantum gravity nature of the noncommutative geometry, as is in the beginning of the Universe [17].

${ }^{11}$ Particles may tunnel quantum mechanically, by their wave nature also. 
non-rotating case $\left(r_{-}=0\right)$, the system is quite normal and the Killing and apparent horizons coincide, except the shift of the horizon in (18). In this case, the Hawking temperature can be defined as usual with a constant shift ${ }^{12}: \hat{T}_{H}=\left(\hat{r}_{+}+l \theta c_{\phi} / 2\right) /\left(2 \pi l^{2}\right)$.

However, the (near horizon) geometry spoils the regularity in general since, as $\eta \rightarrow$ 0, the radial coordinate $\eta$ becomes time-like and the Euclidean procedure itself becomes invalid. Thus, the conventional way of defining the temperature is not valid anymore and this might be a general phenomena for the noncommutative geometry with the smeared horizons. Somehow we suspect that $T_{H}=\tilde{\kappa} / 2 \pi$, which converges to the usual Hawking temperature $T_{H}=\kappa_{+} / 2 \pi$ for the commutative case, can represent the characteristic of the thermodynamical temperature of the smeared systems. The details of this definition are beyond the scope of this paper.

5. The origin $r=0$ is also smeared by a "new" horizon for $\theta c_{\phi}>0$ : There is a third solution of $\hat{N}^{2}=0$ at

$$
\hat{r}_{--}=l \theta c_{\phi}
$$

when $\theta c_{\phi}>0$. This provides a new "apparent horizon" ${ }^{13}$, inside the inner horizon, encircling the origin. The interior region of $\hat{r}_{--}$has two-times as in the region between $\tilde{r}_{+}$and $\hat{r}_{+}$.

In the pure $A d S_{3}$ solutions or the negative mass black holes, generally, which can be obtained by considering $r_{ \pm}^{2} \rightarrow-r_{ \pm}^{2}$ in the black hole solution (12), there is no event horizon and there appears a naked conical singularity at the origin. The conical singularity may be smeared by this new horizon as the results of the spatial noncommutativity

It is also noted that the appearance of the new horizon near the origin depends on the sign of $\theta c_{\phi}$, which is analogous to the sign dependence on the existence of soliton solutions, "fluxons" in the field theories [3]].

\section{Acknowledgments}

We would like to thank Hyun Seok Yang for helpful discussions. This work was supported by the Korea Research Foundation Grant funded by Korea Government(MOEHRD) (KRF2007-359-C00011;M.-I.P.) (KRF-2005-075-C00009;H.-C.K.) and in part by the Korea Science and Engineering Foundation Grant No. R01-2004-000-10526-0 and through the Center for Quantum Spacetime (CQUeST) of Sogang University with grant number R11-2005-021.

[1] H. J. Groenewold, Physica 12, 405 (1946); J. E. Moyal, Proc. Cambridge Phil. Soc. 45, 99 (1949).

[2] A. Connes, "Noncommutative Geometry", Academic Press (1994); A. Connes, M. R. Douglas, and A. S. Schwarz, JHEP 02, 003 (1998).

${ }^{12}$ If one considers the Bekenstein-Hawking entropy [20], $\hat{S}_{B H}=2 \pi \hat{r}_{+}$with respect to the horizon $\hat{r}_{+}$, one would have the first law of thermodynamics with the black hole mass $\hat{M}=\left(\hat{r}_{+}^{2}+l \theta c_{\phi} \hat{r}_{+}\right) / 2 l^{2}$, up to some additive constant terms. But, it is not clear whether the Bekenstein's area law is satisfied even in the noncommutative geometry, which can be regarded as the higher derivative gravities [16].

${ }^{13}$ Similar situation also occurs in AdS black holes with self-interacting scalar hairs [21]. 
[3] M. R. Douglas and N. A. Nekrasov, Rev. Mod. Phys. 73, 977-1029, (2001), and references therein.

[4] A. Pinzul and A. Stern, Class. Quant. Grav. 23, 1009 (2006).

[5] A. Achucarro and P. K. Townsend, Phys. Lett. B 180, 89 (1986); E. Witten, Nucl. Phys. B 311, 46 (1988).

[6] M. Banados, O. Chandia, N. E. Grandi, F. A. Schaposnik and G. A. Silva, Phys. Rev. D 64, 084012 (2001); S. Cacciatori, D. Klemm, L. Martucci and D. Zanon, Phys. Lett. B 536, 101 (2002); S. Cacciatori and L. Martucci, Phys. Lett. B 542, 268 (2002).

[7] N. Grandi and G. A. Silva, Phys. Lett. B 507, 345 (2001).

[8] E. Witten, Commun. Math. Phys. 121, 351 (1989).

[9] O. Coussaert, M. Henneaux, and P. van Driel, Class. Quant. Grav. 12, 2961 (1995); P. Oh and M. I. Park, Mod. Phys. Lett. A 14, 231 (1999); M.-I. Park, Nucl. Phys. B 544, 377 (1999).

[10] N. Seiberg and E. Witten, JHEP 09, 032 (1999).

[11] L. Bonora, M. Schnabl, M. M. Sheikh-Jabbari and A. Tomasiello, Nucl. Phys. B 589, 461 (2000).

[12] A. P. Polychronakos, JHEP 11, 008 (2000).

[13] M. Banados, C. Teitelboim, and J. Zanelli, Phys. Rev. Lett. 69, 1849 (1992).

[14] S. Carlip, J. Gegenberg, and R. B. Mann, Phys. Rev. D 51, 6854 (1995).

[15] A. H. Chamseddine, Commun. Math. Phys. 218, 283 (2001); Phys. Lett. B 504, 33 (2001); J. W. Moffat, Phys. Lett. B 491, 345 (2000).

[16] M.-I. Park, Phys. Lett. B 647, 472 (2007); Phys. Rev. D 77, 026011 (2008); Phys. Rev. D 77, $126012(2008)$.

[17] J. B. Hartle and S. W. Hawking, Phys. Rev. D 28, 2960 (1983).

[18] M. K. Parikh and F. Wilczek, Phys. Rev. Lett. 85, 5042 (2000).

[19] J. B. Hartle and S. W. Hawking, Phys. Rev. D 13, 2188 (1976).

[20] J. D. Bekenstein, Phys. Rev. D 7, 2333 (1973); S. W. Hawking, Phys. Rev. Lett. 26, 1344 (1971).

[21] C. Martinez, J. P. Staforelli, and R. Troncoso, Phys. Rev. D 74, 044028 (2006); M.-I. Park, Phys. Lett. B 597, 237 (2004). 